\section{Diffraction from Dislocations}

WILsON ${ }^{1}$ has calculated the nature of the diffraction from a cylindrical crystal with a single axial screw dislocation. Diffraction from an edge dislocation is more difficult to treat theoretically, because of the more complex nature of the elastic field surrounding the dislocation. An approximate treatment has been given by Wilson ${ }^{2}$, who assumed that the displacements of the atoms were parallel to the Burgers vector. We have succeeded in solving the diffraction problem for a cylindrical crystal with an axial edge dislocation, in which the displacements correspond to isotropic elastic theory. Two independent methods were used: straightforward calculation (by 'T. S.), and direct observation in a Lipson diffractometer of the optical diffraction pattern of a two-dimensional grating representing the crystal (B.T.M. W.). In Fig. 1 we reproduce the optical diffraction pattern given by a grating representing (a) a perfect crystal (no dislocation) and $(b)$ a simple-cubic crystal with an edge dislocation normal to the plane corresponding to the plane of the grating.

Our results can be summarized as follows. The $h$-axis in reciprocal space is parallel to the slip direction (Burgors vector) and the $l$-axis to the axis of the dislocation. (1) Any spreading of the diffracted intensity in reciprocal space is confined to the planes $l=0, \pm 1 \ldots$, and the intensity within these planes is independent of $l$. (2) The hol reflexion is broader and weaker than the $0 k l$ reflexion for equal values of $h$ and $k$. (3) The maxima of the $h 0 l$ reflexion are displaced from the ideal reciprocal lattice point by an amount that increases linearly with $h$; the maxima of the $0 k l$ reflexion are also displaced, but the displacement is roughly independent of $k$ for small $k$. (4) The intensity at the ideal $h 0 l$ and $0 k l$ lattice points is zero for $h, k>4$. (5) The form of the distribution of intensity around an ideal reciprocal lattice point depends on its co-ordinates in reciprocal space, on the strength of the dislocation and on the value of Poisson's ratio, but not otherwise on the crystal structure. In contrast with the case of a screw dislocation ${ }^{1}$, the spread of intensity around

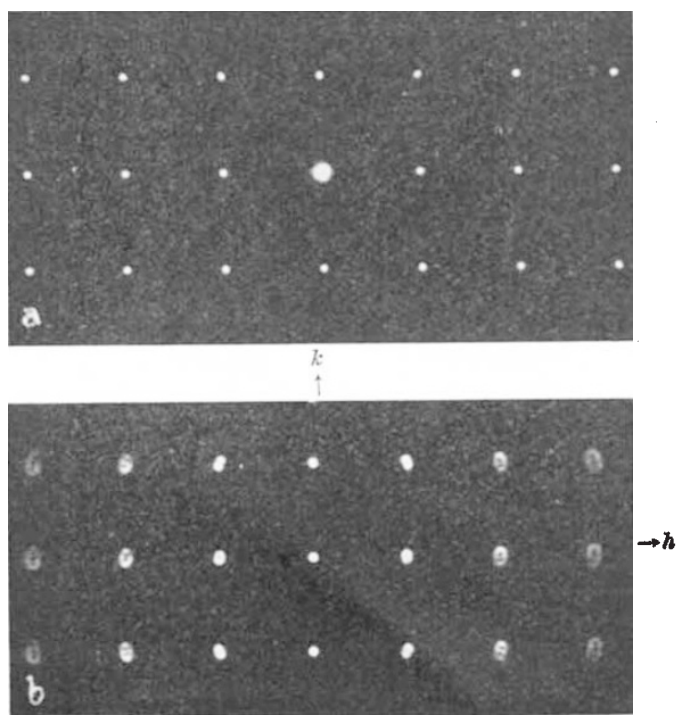
Fig. 1. Diffraction patterns from gratings representing ( $a$ ) perfect
srystal, $(b)$ crystal with dislocation. The horizontal axis in $(b)$ corresponds with the slip direction the ideal reciprocal lattice point is not of circular symmetry.

These results were obtained using an average value of 0.3 for Poisson's ratio. It is clear that the diffraction pattern is quite different from that proposed by Wilson on the basis of the more approximate theory ${ }^{2}$.

Our thanks are due to Prof. F. C. Frank for discussion of this problem, and to Dr. J. Thewlis, who suggested the use of the optical method for its solution.

$$
\text { T. SUzukI }
$$

H. H. Wills Physical Laboratory, Royal Fort, Bristol 8.

\section{B. T. M. Willis}

Atomic Energy Research Establishment, Harwell,

Nr. Didcot, Berks.

Dec. 13.

${ }^{1}$ Wilson, A. J. C., Acta Cryst., 5, 318 (1952). ${ }^{2}$ Wilson, A. J. C., Research, 3, $387(1950)$.

\section{Galvanic Measurement of Dissolved Oxygen}

AN exceedingly sensitive static $^{1}$ as well as continuous ${ }^{2}$ method for determining molecular oxygen in the gas phase has been described by Hersch. This discovery has led to the development of a reliable commercial instrument for the continuous indication and recording of oxygen in gases. Hersch has also proposed $\mathrm{d}^{2}$ the application of this method to oxygen dissolved in boiler water, effluents, biofluids, and fermentation media by driving the oxygen into an inert carrier.

The measurement of oxygen in an aqueous phase by polarographic or amperometric means has been often described, but the sensitivity of these methods is limited by the value of the oxygen diffusion current. Moreover, reducible constituents other than oxygen can interfere, while salt deposits and adsorbable organic material progressively block the cathode ${ }^{3}$. The application of the Hersch galvanic system to liquids in the way suggested would largely eliminate these drawbacks.

We have experimented with a simple apparatus designed to adapt the method described by Hersch to the measurement of dissolved oxygen, which has proved effective and reliable.

We used demineralized water 'scrubbed' by means of hydrogen passed via a 'Deoxo' purifier through a sintered glass disk at the bottom of a reservoir. The water is fed, at constant flow-rate determined by a static head, to a glass vessel having an outlet at the bottom into a vertical tube via a constriction constructed in such a way that a vortex is formed. The vessel contains oxygen-free hydrogen which is sucked into the vortex and flows in a series of bubbles with the water down the vertical tube, taking from the water a proportion of the dissolved oxygen. We have found it surprisingly easy to establish conditions in which the proportion of oxygen transferred to the hydrogen in this 'oxygen exchange column' remains constant. At the bottom of the column, the water/ hydrogen mixture runs into a vessel of larger diameter where the water separates from the gas and flows 